\title{
Solution structure and mutagenesis of the caspase recruitment domain (CARD) from Apaf-1
}

\author{
CL Day ${ }^{\star, 1}$, C Dupont ${ }^{1}$, M Lackmann², DL Vaux ${ }^{3,4}$ and \\ MG Hinds ${ }^{4,5}$ \\ 1 Institute of Molecular BioSciences, Massey University, Private Bag 11222 , \\ Palmerston North, New Zealand \\ 2 Ludwig Institute for Cancer Research, Post Office, Royal Melbourne Hospital, \\ Parkville 3050, Australia \\ 3 The Walter and Eliza Hall Institute of Medical Research, Post Office, Royal \\ Melbourne Hospital, Parkville 3050, Australia \\ 4 The Cooperative Research Centre for Cellular Growth Factors \\ ${ }^{5}$ Biomolecular Research Institute, 343 Royal Parade, Parkville 3052, Australia \\ * Corresponding author: CL Day, Institute of Molecular BioSciences, Massey \\ University, Private Bag 11 222, Palmerston North, New Zealand. \\ Tel: +64 63505515 extn 2564; Fax: +64 6350 5688; \\ E-mail: C.L.Day@massey.ac.nz
}

Received 30.7.99; accepted 16.8.99

Edited by G Melino

\begin{abstract}
Activation of procaspase-9, a key component of the apoptosis mechanism, requires the interaction of its caspase recruitment domain (CARD) with the CARD in the adaptor protein Apaf-1. Using nuclear magnetic resonance spectroscopy and mutagenesis we have determined the structure of the CARD from Apaf-1 and the residues important for binding the CARD in procaspase-9. Apaf-1's CARD contains seven short $\alpha$ helices with the core six helices arranged in an antiparallel manner. Residues in helix 2 have a central role in mediating interaction with procaspase-9 CARD. This interaction surface is distinct from that proposed based on the structure of the CARD from RAIDD, but is coincident with that of the structurally similar FADD death effector domain and the Apaf-1 CARD interface identified by crystallographic studies.
\end{abstract}

Keywords: apoptosis; CARD interactions; caspase; NMR spectroscopy; protein structure

Abbreviations: CARD, caspase recruitment domain; DD, death domain; DED, death effector domain; DTT, dithiothreitol; GST glutathione-S-transferase; NMR, nuclear magnetic resonance; PBS, phosphate buffered saline; r.m.s.d., root mean square difference

\section{Introduction}

Apoptosis, or programmed cell death, is the physiological process that brings about the removal of unwanted or damaged cells from an organism. ${ }^{1}$ The key effector proteins of apoptosis are a family of cysteine aspartic proteases termed caspases. In healthy cells procaspases exist as catalytically inactive zymogens. Based on the substrate specificity of each caspase and its prodomain structure, a hierarchical cascade has been proposed whereby upstream initiator caspases, with long prodomains, activate downstream caspases, that have short prodomains. ${ }^{2}$ While this cascade accounts for the activation of all downstream or executioner caspases such as caspase-3, initiator caspases such as caspase- 8 and caspase- 9 are activated by adaptor proteins that interact with their prodomains. $^{3}$

Initiator caspases have large prodomains that contain either a caspase recruitment domain (CARD) or death effector domain (DED). Like the structurally related death domains (DDs), DEDs and CARDs function as interaction motifs that bring about the specific association of two proteins with complementary domains. ${ }^{4}$ For example, interaction of procaspase-8 with the adaptor FADD is mediated by DEDs in both proteins, ${ }^{5}$ while CARDs mediate association of the adaptor proteins Apaf- 1 and RAIDD with the prodomains of caspase- 9 and caspase-2, respectively. ${ }^{6,7}$

Apaf-1 and caspase-9 are key, non-redundant, mediators of apoptosis, since mice in which either is deleted show dramatic phenotypic changes in their central nervous systems and cells from these mutant animals have defects in their apoptotic responses to a variety of agents. ${ }^{8-11}$ Elucidating how they interact will be central to understanding their roles in apoptosis.

Based on sequence similarity, Apaf- 1 can be divided into three domains. The CARD is found in the N-terminal $\sim 100$ residues, and allows interaction with the CARD of procaspase- $9 .^{7-12}$ The succeeding $\sim 300$ residues resemble the analogous region in CED4, an adaptor protein from Caenorhabditis elegans, and encode a domain with a nucleotide-binding $\mathrm{P}$-loop motif. The large $\mathrm{C}$-terminal region ( $\sim 800$ residues) of Apaf-1 encodes twelve WD-40 repeats that share no significant sequence similarity with CED4. ${ }^{13}$

Structures determined for one DED and several DDs and CARDs reveal that all have a similar three-dimensional fold comprised of six $\alpha$-helices organized in an antiparallel arrangement. ${ }^{14-19}$ However, the details of helix packing, and the surface residues critical for protein-protein interactions differ. Hydrophobic residues in helices 2 and 5 of the DED in the adaptor protein, FADD, have been proposed to mediate association with DEDs in the prodomain of caspase- $8,{ }^{17}$ while electrostatic interactions have been shown to mediate oligomerization of both DDs and CARDs. ${ }^{18,19}$

We report here the solution structure of the CARD from Apaf-1 and mutagenesis data that identifies helix 2 as critical for interaction with the CARD from procaspase-9. While this manuscript was in preparation the crystal structures of Apaf-1 CARD alone and in complex with the prodomain of caspase- 9 appeared. ${ }^{19}$ Except for small differences in helix length, the solution structure of Apaf-1 CARD is very similar to the structure determined by 
crystallographic methods. Our mutagenesis data support the mechanism of interaction of procaspase-9 CARD and Apaf-1 reported by Qin et al. ${ }^{19}$ and suggest that the general model for CARD interactions proposed by Chou et al. ${ }^{16}$ is incorrect, but indicate that some CARDs and DEDs use similar surfaces to mediate domain interaction.

\section{Results}

\section{Structure determination}

The region of human Apaf-1 (residues 1-97) containing the CARD (Figure 1A) was over-expressed in E. coli and shown to be $\alpha$-helical by circular dichroism spectroscopy (data not shown). This protein had very good solution properties at $\mathrm{pH} 6.7$ and was stable at $1.5 \mathrm{mM}$ for several months, allowing the structure to be determined using triple resonance multidimensional nuclear magnetic resonance (NMR) spectroscopy. ${ }^{20}$ Resonance assignments of both backbone and side chain atoms were essentially complete apart from the resonances of Ser23 and Ser96 which could not be assigned. Five $\mathrm{N}$-terminal residues from the expression vector (residues -5 to -1 ) were included in the calculation although they have been omitted from the figures.

The structures are well ordered (Table 1) and have good stereochemical properties, with over $98 \%$ of backbone angles falling in the allowed regions of the Ramachandran plot and a pairwise root mean square difference (r.m.s.d.) of $0.50 \pm 0.08 \AA$ over the backbone atoms $\left(\mathrm{N}, \mathrm{C}^{\alpha}, \mathrm{C}\right)$ of helical residues. The precision of the structures is also apparent from Figure 1B where the superimposed backbones of residues 1-92 and side chains of the buried residues are shown.

\section{Structure description}

Apaf-1 CARD (Figure 1B-D) consists of seven $\alpha$-helices (residues 3-10, 13-19, 24-32, 37-45, 51-61, 66-77, $81-88$ ) closely packed around a hydrophobic core comprised of many of the conserved hydrophobic residues found in most CARDs. The solution structure of Apaf-1 CARD is similar to the crystal structures of both Apaf-1 CARD and the prodomain of caspase-9. ${ }^{19}$ Unlike other six-helix bundle death domain structures, helix $\mathrm{H} 1$ is shorter and an additional short helix, $\mathrm{H}^{1}$ (residues 13-19 shown in yellow in Figure $1 \mathrm{C}, \mathrm{D}$ ), connects helices $\mathrm{H} 1$ and $\mathrm{H} 2$ in Apaf-1 CARD. The break in helix $\mathrm{H} 1$ at $\mathrm{His} 12$ is indicated by the presence of a non-helical coupling constant $(8.9 \mathrm{~Hz})$ and a change in the chemical shift index from that expected for a helical residue.

Like other six-helix bundle death domain structures the core six helices in the solution structure of Apaf-1 CARD are arranged in an antiparallel manner that places the $\mathrm{N}$ and $\mathrm{C}$-termini of the molecule in close proximity (Figure 1C). Helices $\mathrm{H} 1, \mathrm{H} 2, \mathrm{H} 3$ and $\mathrm{H} 6$ are located on the ends of the molecule and are amphipathic, while helices $\mathrm{H} 4$ and $\mathrm{H} 5$ occupy a more central position and have very few solvent accessible residues. The buried nature of helices $\mathrm{H} 4$ and $\mathrm{H} 5$ and their relative rigidity is indicated by both the $\mathrm{NH}$ exchange and $\left\{{ }^{1} \mathrm{H}\right\}^{15} \mathrm{~N}$ heteronuclear NOE data (data not shown). Of the $43 \mathrm{NHs}$ that did not exchange immediately at $30^{\circ} \mathrm{C}, 19$ are located either in helices $\mathrm{H} 4$ and $\mathrm{H} 5$ or the short loop that connects them. All of the remaining slowly exchanging backbone $\mathrm{NHs}$ are located within helices or at helix termini.

The surface of Apaf-1 CARD is highly charged (Figures $1 \mathrm{~A}$ and 2). The most distinctive feature is the basic patch formed by residues Arg6, Lys42, Lys58, Lys62 and Lys63. Another area of basic potential comprises Lys18, Lys21, Arg44 and Arg52. The acidic residues are more evenly distributed over the surface of the protein (Figure 2). There is no extensive hydrophobic area on the surface of Apaf-1 CARD.

\section{Comparison of Apaf-1 CARD with RAIDD CARD and FADD DED}

The CARD of Apaf- 1 and that of RAIDD ${ }^{16}$ superimpose with a r.m.s.d. of $2.1 \AA$ for 45 equivalent $\alpha$-carbons chosen on the basis of a structural alignment. ${ }^{21}$ Helices $\mathrm{H} 2, \mathrm{H} 3, \mathrm{H} 5$ and $\mathrm{H} 6$ occupy similar positions in both proteins and overlay quite closely although the length of helices $\mathrm{H} 5$ and $\mathrm{H} 6$ differs. Helix $\mathrm{H} 5$ is longer in Apaf-1 CARD while helix $\mathrm{H} 6$ is shorter and the C-terminus of Apaf-1 CARD has an irregular conformation (Figures $1 \mathrm{~A}$ and $3 \mathrm{~A}$ ). The differences in length and conformation of helix $\mathrm{H} 5$ and the following loop region may be linked to the two amino acid deletion seen in RAIDD CARD (Figure 1A).

The main differences between the two CARD structures are found in the position and conformation of helix $\mathrm{H} 1$ and the loop that connects helices $\mathrm{H} 1$ and $\mathrm{H} 2$ (Figure 3A). The $\mathrm{H} 1-\mathrm{H} 2$ loop is poorly defined in RAIDD CARD, this may reflect either an intrinsic mobility or a lack of NMR constraints in this region of the molecule. In contrast, helix $\mathrm{H} 1$ is well defined in both structures and the change in length and position of this helix represents a significant difference. The orientation of helix $\mathrm{H} 4$ is also different in the two structures. In RAIDD CARD, helices $\mathrm{H} 4$ and $\mathrm{H} 5$ are approximately parallel while in Apaf-1 CARD the $\mathrm{N}$ terminus of helix $\mathrm{H} 4$ is displaced outward from the core of the molecule (Figure $3 \mathrm{~A}$ ). The displacement in Apaf-1 CARD is likely to be related to the altered conformation of the loop that connects helices $\mathrm{H}^{\prime}$ and $\mathrm{H} 2$, as the buried residues in this loop pack between helices $\mathrm{H} 4$ and $\mathrm{H} 5$ (Figure 1B).

Unlike RAIDD CARD and other death domain structures but similar to the CARD crystal structures, ${ }^{19}$ Apaf-1 CARD has an additional short helix, $\mathrm{H}^{\prime}$, that lies almost perpendicular to $\mathrm{H} 1\left(\mathrm{H} 1-\mathrm{H} 1^{\prime}\right.$ inter-helical angle $\left.71 \pm 4^{\circ}\right)$. The altered conformation of the $\mathrm{H} 1-\mathrm{H} 2$ loop in Apaf-1 CARD results in differences in both the solvent accessibility and packing of residues 18-27 relative to RAIDD CARD. In particular, Ala15, Ile20 and Thr22 are buried from solvent while Lys21 and Ser23 are only partially accessible in Apaf1 CARD. The corresponding residues in RAIDD CARD are more solvent accessible (Figure 1). As a consequence of these changes to the $\mathrm{H} 1-\mathrm{H} 2$ loop, Apaf-1 CARD does not have an extended hydrophobic patch on the surface.

Comparison with other six-helix bundle death domain structures, for which the coordinates are available, reveals that Apaf-1 CARD is most similar to the structure of the 
A
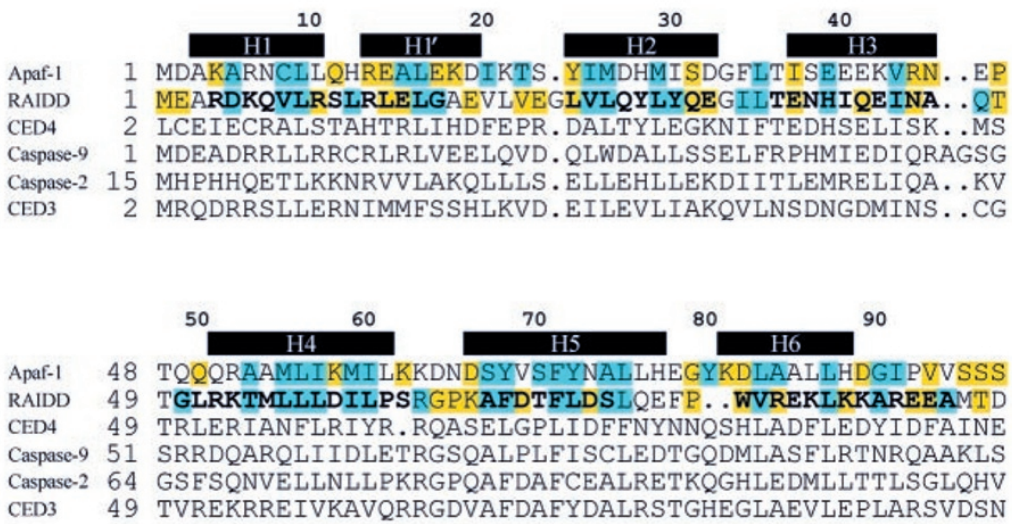

B

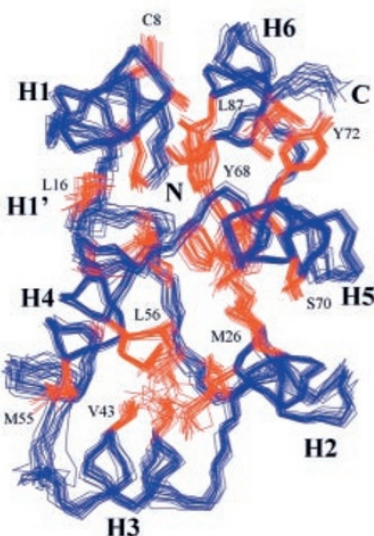

H3

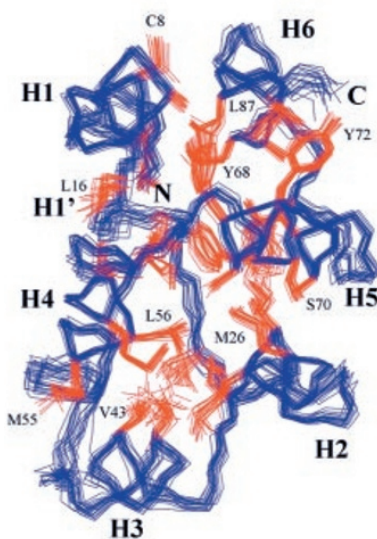

$\mathrm{H3}$

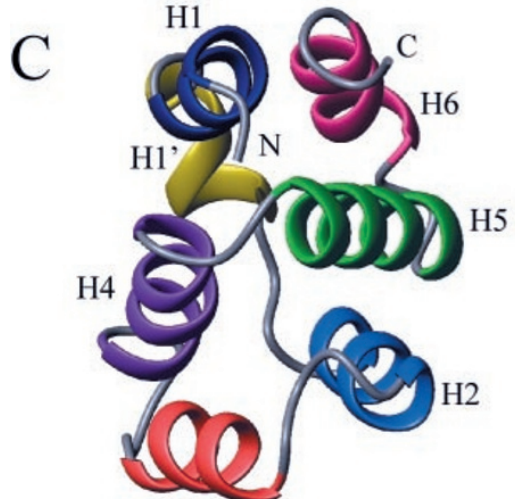

$\mathrm{H} 3$

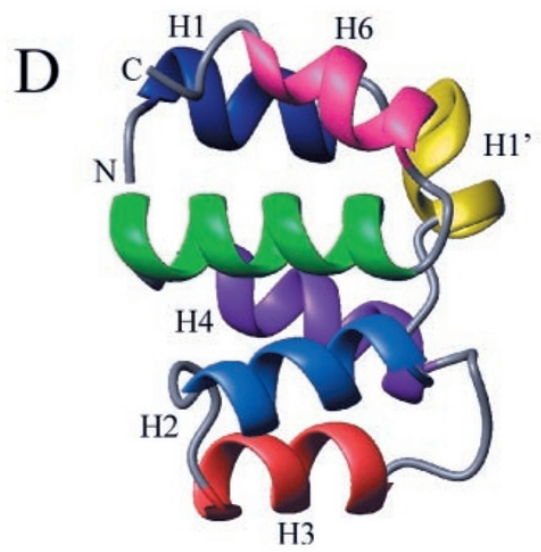

Figure 1 Structure of Apaf-1 CARD. (A) Sequence alignment of the CARDs from Apaf-1, RAIDD, CED4, Caspase-9, Caspase-2 and CED3 (Genbank accession numbers AF013263; U79115; X69016; U60521; U13021; L29052, respectively). Residues buried (<15\% solvent accessible) within the hydrophobic core are shown in blue and exposed residues (>38\% solvent accessible) are in yellow for both Apaf-1 CARD and RAIDD CARD. The $\alpha$ helices in Apaf-1 CARD are indicated by a black bar above the sequences and are shown in bold type for RAIDD CARD. The buried and accessible residues for RAIDD CARD were determined from the pdb file (accession number $3 \mathrm{crd}$ ). (B) Stereoview of the backbone (N, $\left.\mathrm{C}^{\alpha}, \mathrm{C}\right)$ traces (blue) of the 20 lowest energy structures for Apaf-1 CARD superimposed over the backbone atoms $\left(N, C^{\alpha}, C\right)$ of residues 1-95 (residues 1-92 are shown). The side chains of the buried residues are in orange. (C) End on view of a ribbon depiction of the NMR structure closest to the geometric average of Apaf-1 CARD (residues 1-92 are shown). The helices and position of the $\mathrm{N}$ - and $\mathrm{C}$-termini are labelled. (D) Orthogonal view of Figure $1 \mathrm{C}$. This view highlights the linking position of helix $\mathrm{H}^{\prime}$ 
Table 1 Structural statistics for the 20 lowest energy structures of Apaf-1 CARD

\begin{tabular}{|c|c|c|}
\hline \multicolumn{3}{|l|}{ Experimental constraints } \\
\hline Total distance constraint & \multicolumn{2}{|c|}{2376} \\
\hline Sequential $(|i-j|=1)$ & \multicolumn{2}{|c|}{555} \\
\hline Short-range $(1<|i-j|<5)$ & \multicolumn{2}{|c|}{706} \\
\hline Long-range $(|i-j| \geqslant 5)$ & \multicolumn{2}{|c|}{552} \\
\hline Intra-residue & \multicolumn{2}{|c|}{501} \\
\hline Hydrogen bonds ${ }^{a}$ & \multicolumn{2}{|c|}{31} \\
\hline Total angle constraints $\left(85 \phi ; 71 \Psi ; 17 \chi_{1}\right)$ & \multicolumn{2}{|c|}{173} \\
\hline $\begin{array}{l}\text { r.m.s.d. from experimental distance } \\
\text { restraints }(\AA)(2376)\end{array}$ & \multicolumn{2}{|c|}{$0.0304+0.001$} \\
\hline $\begin{array}{l}\text { r.m.s.d. from experimental dihedral } \\
\text { restraints }\left({ }^{\circ}\right)(173)\end{array}$ & \multicolumn{2}{|c|}{$0.30 \pm 0.11$} \\
\hline r.m.s.d. from idealized covalent geometry & \multirow{2}{*}{\multicolumn{2}{|c|}{$00049+001$}} \\
\hline Bonds $(\AA)$ & & \\
\hline Angles $\left({ }^{\circ}\right)$ & \multirow{2}{*}{\multicolumn{2}{|c|}{$\begin{array}{l}0.65 \pm 0.02 \\
0.62 \pm 0.03\end{array}$}} \\
\hline Impropers $\left({ }^{\circ}\right)$ & & \\
\hline \multicolumn{3}{|l|}{ Measures of structural quality } \\
\hline \multicolumn{3}{|c|}{ PROCHECK ${ }^{\mathrm{b}} \%$ residues in region of Ramachandran plot } \\
\hline Most favorable region & \multicolumn{2}{|c|}{83.8} \\
\hline Additionally allowed & \multicolumn{2}{|c|}{14.6} \\
\hline Generously allowed & \multicolumn{2}{|c|}{1.1} \\
\hline Disallowed & \multicolumn{2}{|c|}{0.5} \\
\hline Angular order. Residues with $\mathrm{S}(\phi) \geqslant 0.9$; & \multirow{2}{*}{\multicolumn{2}{|c|}{$\begin{array}{l}100 \\
101\end{array}$}} \\
\hline Residues with $S(\Psi) \geqslant 0.9$ & & \\
\hline \multicolumn{3}{|l|}{ Mean pairwise r.m.s.d. $(\AA)$} \\
\hline $\begin{array}{l}\text { Residues superimposed } \\
\text { All }\end{array}$ & $\begin{array}{r}\mathrm{N}, \mathrm{C}^{\alpha}, \mathrm{C} \\
1.98 \pm 0.46\end{array}$ & $\begin{array}{l}\text { All heavy } \\
2.24 \pm 0.34\end{array}$ \\
\hline $1-95$ & $0.90 \pm 0.46$ & $1.49 \pm 0.18$ \\
\hline Helices $(\mathrm{H} 1-\mathrm{H} 6)$ & $0.50 \pm 0.08$ & $1.28 \pm 0.10$ \\
\hline
\end{tabular}

${ }^{\text {a }}$ Two distances constraints per hydrogen bond were applied. bPROCHECK_NMR analysis ${ }^{33}$

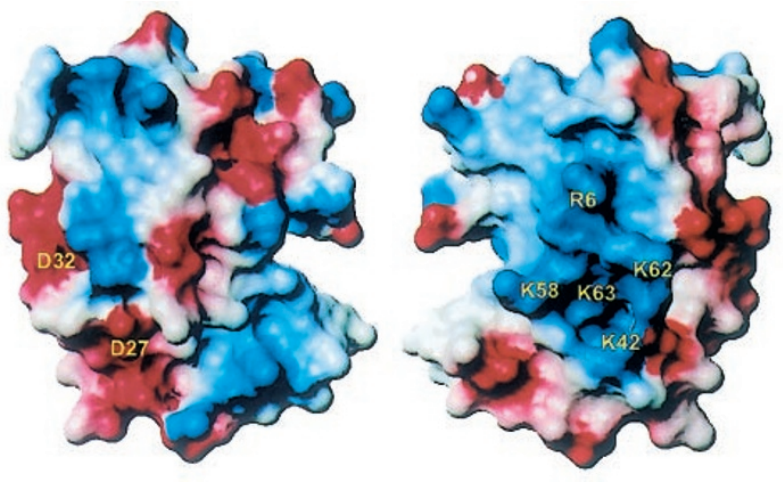

Figure 2 Surface characterization of Apaf-1 CARD. Electrostatic molecular surface rendering of the NMR structure closest to the geometric average of Apaf-1 CARD (residues 1-95). Surfaces are colored by electrostatic charge, with regions of negative potential red and those with positive potential blue. The right view is in a similar orientation to the ribbon diagram in Figure 1D and the left view is rotated $180^{\circ}$ about the vertical axis

DED from FADD, ${ }^{17}$ with a r.m.s.d. of $1.8 \AA$ over $48 \alpha$ carbon atoms (Figure 3B). Helices $\mathrm{H} 2, \mathrm{H} 3, \mathrm{H} 5$ and $\mathrm{H} 6$ have very similar positions in both structures although the length varies. The main differences between the two structures lie in the loop that connects helices $\mathrm{H} 4$ and $\mathrm{H} 5$ and the position of helix $\mathrm{H} 1$.

\section{Binding of Apaf-1 CARD to procaspase-9 CARD}

Previous studies suggested that the interaction between Apaf- 1 and procaspase- 9 is mediated by the CARDs in each protein. $^{6,12}$ To demonstrate a direct interaction between the CARDs, and to allow the residues in Apaf-1 CARD that mediate this interaction to be mapped, we expressed the CARD from procaspase-9 (residues 1-99) in E. coli. Purified procaspase- 9 CARD fused to GST and bound to resin could specifically pull down purified Apaf-1 CARD, however, no Apaf-1 CARD was precipitated when GST alone was on the resin (data not shown). In addition, no interaction between either procaspase-9 CARD and GST/procaspase-9 CARD or Apaf-1 CARD and GST/Apaf-1 CARD was seen (data not shown) indicating that no homo-oligomers formed. The role of a number of residues on the surface of Apaf-1 CARD was then evaluated using alanine scanning mutagenesis (Table 2). ${ }^{22}$

Initially, residues that were predicted to mediate interactions with procaspase-9 CARD ${ }^{16}$ and which form a basic patch on Apaf-1 CARD (K42, K58, K62, K63) were mutated (Figure 2). When mutated individually or as pairs, no reduction was seen in the amount of procapsase- 9 CARD precipitated by any of these mutants relative to wild type Apaf-1 CARD (Table 2). In contrast, a series of mutations to solvent accessible residues on the opposite face (Y24A, D32A, D27A, N73A) decreased the ability of Apaf-1 CARD to precipitate procaspase-9 CARD (Table 2 and Figure 4). Comparison of ${ }^{15} \mathrm{~N}-\mathrm{HSQC}$ spectra from the mutant Y24A and wild type Apaf-1 CARD showed that only a few resonances of $\mathrm{NHs}$ near the site of mutation were altered significantly, indicating the overall fold of Apaf-1 CARD was not disrupted. This implies that the Y24A mutation directly disrupts procaspase-9 CARD binding to Apaf-1 CARD. As Asp27, Asp32 and Asn73 are all part of the extended surface formed by the exposed residues from helices $\mathrm{H} 2$ and $\mathrm{H} 5$ (Figure 4), it is likely that these residues also participate in binding procaspase-9 CARD. As well as the mutations to residues in the basic patch a number of other mutations to surface residues (K18A, K21A, Q49A, K81A, D89A) did not disrupt the interaction between the two CARDs, suggesting that the interaction interface is confined to one face of the molecule.

\section{Discussion}

Six-helix bundle death domains are the key determinants of oligomerization in many proteins involved in regulating apoptosis and understanding the molecular basis of oligomerization specificity is central to understanding the apoptotic process. The solution structure of Apaf-1 CARD described here is consistent with the crystal structures reported for Apaf-1 CARD in isolation and in complex with the prodomain of caspase-9. ${ }^{19}$ These structures display many differences from the structure of RAIDD CARD. ${ }^{16}$ In particular, helix $\mathrm{H} 1$ is shorter and shifted relative to the corresponding helix in RAIDD, and helices $\mathrm{H} 1$ and $\mathrm{H} 2$ are linked by an additional helix, $\mathrm{H}_{1}$ '. Other differences include the orientation of helix $\mathrm{H} 4$ and the length of both helices $\mathrm{H} 5$ and $\mathrm{H} 6$. Although the fold is similar these changes cause differences in the solvent accessible residues between the two CARDs that 

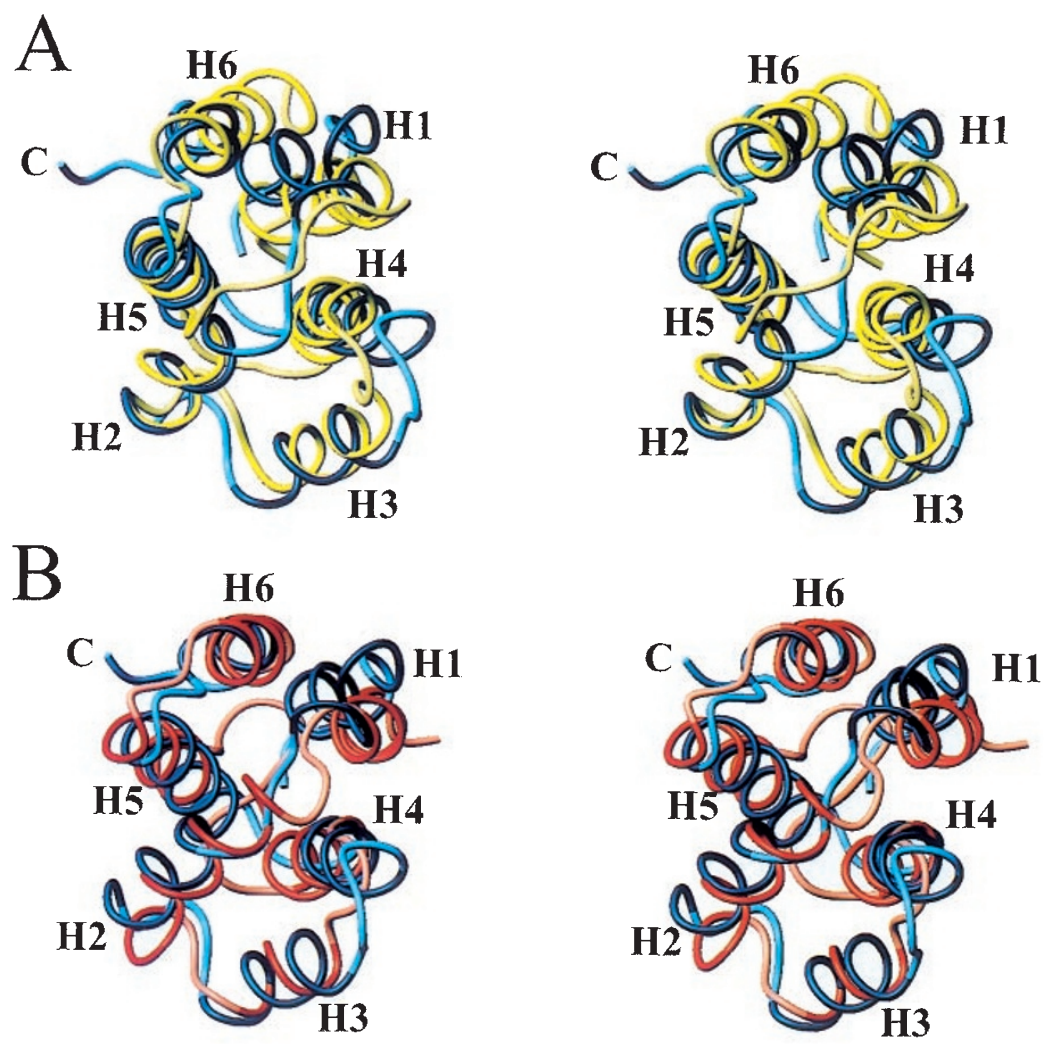

Figure 3 Stereoview superposition ribbon diagrams comparing Apaf-1 with RAIDD CARD and FADD DED. In both cases the residues used to overlay the two structures were identified using the 3D structure comparison program TOP. ${ }^{21}$ (A) The helices in Apaf-1 CARD are shown in dark blue and the connecting residues in light blue while the helices in RAIDD CARD are shown in dark yellow and the connecting loops in light yellow. $C^{\alpha}$ atoms of residues 25-40, 43-45, 56-62, 6365, 67-75 and 82-88 from Apaf-1 CARD and 26-41, 44-46, 57-63, 65-67, 68-76 and 81-87 from RAIDD CARD (pdb accession 3crd) were superimposed. (B) Apaf-1 is colored as above. Helices in FADD DED are colored dark orange and the loops light orange. $\mathrm{C}^{\alpha}$ atoms of residues $16-18,22-30,35-43,57-61$, 69-90 from Apaf-1 CARD and 12-14, 10-28, 31-39, 43-47, 61-82 from FADD DED (pdb accession 1a1w) were superimposed

Table 2 Analysis of Apaf-1 CARD site specific mutants

\begin{tabular}{lc}
\hline Protein & Binding $^{\mathbf{a}}$ \\
\hline wt Apaf-1 CARD & + \\
K18A/K21A & + \\
Y24A & -- \\
D27A & -- \\
D32A & - \\
F34A & + \\
K42A/K58A & + \\
Q49A & + \\
K62A/K63A & + \\
N73A & --- \\
H77A & + \\
K81A & + \\
D89A & + \\
\hline
\end{tabular}

${ }^{a} A(+)$ indicates that the amount of procaspase-9 CARD bound was indistinguishable from that bound by wild type (wt) Apaf-1 CARD, a (-) indicates that slightly reduced binding was seen, (- -) indicates significantly reduced binding and (- - -) indicates no detectable binding of procaspase-9 CARD

alter the surface properties. Given the low sequence identity of these small domains ( $\sim 20 \%$ across all CARDs), and the variations in helix length, caution must be exercised when

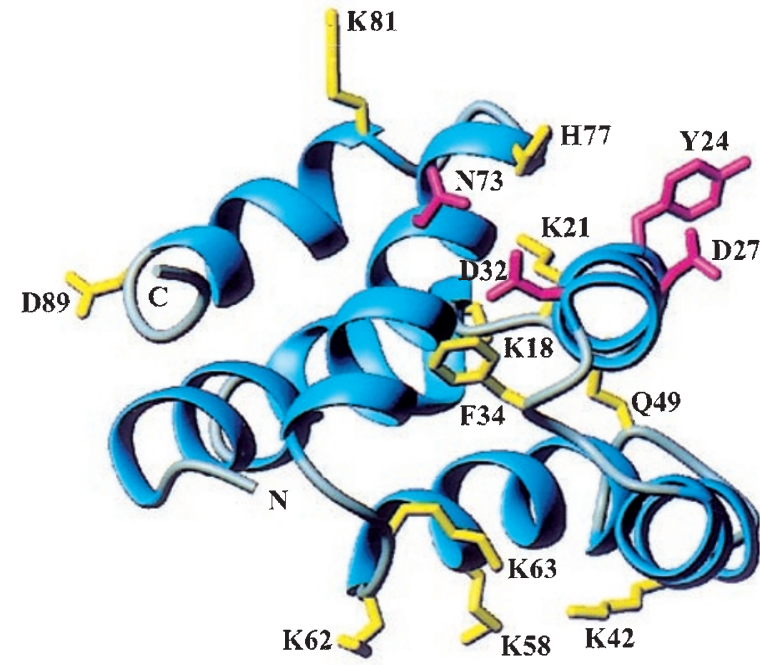

Figure 4 Mapping of mutagenesis data onto the structure of Apaf-1 CARD. Helices 1-7 are in blue, the side chains of residues that decrease binding of Apaf-1 CARD to procaspase- 9 CARD are red and those that have been mutated in this study and have no effect on binding are yellow (residues 1-95 are shown) 
predicting the structure of one CARD based on the structure of another. In particular, the surface features of helices $\mathrm{H} 1$ and $\mathrm{H} 6$ are difficult to predict because of the low level of sequence conservation beyond helix $\mathrm{H} 5$ and the variable orientation of helices $\mathrm{H} 1$ and $\mathrm{H} 6$.

The CARD of Apaf-1 was shown to represent a functionally relevant domain based on its ability to directly and specifically interact with the CARD of procaspase-9. The absence of any self-association of either Apaf-1 CARD or procaspase-9 CARD does not support a role for homooligomerization mediated by CARDs in caspase- 9 activation. Only in the presence of cytochrome $c$ and dATP do Apaf- 1 and procaspase- 9 interact in the cell, suggesting that the interface of Apaf-1 CARD required for procaspase9 CARD binding is either in a different conformation in the absence of cytochrome $c$ and dATP or that it is masked. The structural similarity of Apaf-1 CARD in the bound and free forms ${ }^{19}$ coupled with the observation that deletion of the C-terminal WD-40 domain increases association of Apaf- 1 and procaspase- $9^{12,23}$ suggests the C-terminus plays a role in masking the CARD binding surface in intact Apaf-1.

The surface in Apaf-1 CARD most likely to mediate interaction with procaspase-9 CARD, identified using alanine scanning mutagenesis, includes exposed residues (Tyr24, Asp27, Asp32, Asn73) in helices H2 and H5. This agrees very well with crystallographic data ${ }^{19}$ but is in marked contrast to the model for Apaf-1/procaspase- 9 CARD interactions proposed by Chou et al. ${ }^{16}$ Based on the structure of RAIDD CARD, limited mutagenesis data and homology models of the CARDs from procaspase-2, Apaf-1 and procaspase-9, a general model for CARD/CARD interactions was put forward. The model proposed ${ }^{16}$ that the basic patch formed by helices $\mathrm{H} 3, \mathrm{H} 4$ and the $\mathrm{H} 4-\mathrm{H} 5$ loop (residues 42, 58, 62 and 63) in Apaf-1 CARD would mediate interaction with procaspase-9 CARD. The mutagenesis experiments described do not support a role for these residues in mediating interaction of Apaf-1 CARD with procaspase-9 CARD.

In general the results described here agree well with those of Qin et al. ${ }^{19}$ although several differences do exist. Firstly the crystallographic studies did not implicate helix $\mathrm{H} 5$ in mediating interaction of Apaf-1 CARD with procaspase- 9 CARD although mutation of Asn73 was one of the most disruptive mutations in our investigation. While the side chain of Asn73 is solvent accessible and could conceivably participate in the interface it is also possible that mutation to alanine alters the orientation of helix $\mathrm{H} 2$ therefore disrupting binding. Conversely while the solution structure of Apaf-1 CARD is consistent with an interface role for Helix H3 no mutations reported here address this directly. Mutation of Tyr24, however, indicates that this residue has an important role in binding procaspase-9 CARD. Although not shown in the interface by Qin et al., ${ }^{19}$ it seems likely that it would pack against Arg52 from the prodomain of caspase-9 thereby stabilizing complex formation. In the crystal structure of the complex ${ }^{19}$ Ile30 is part of the interface, however, in the solution structure described here the side chain of lle30 is largely buried and it would require reorientation to allow it to contribute to the interface.
Whether other CARDs use a similar surface to that identified in Apaf-1 CARD to mediate oligomerization remains uncertain, although several results are of interest. Mutagenesis of procaspase-2 identified Asp83 and Glu87 as possible interface residues. ${ }^{6,16}$ Based on sequence alignment, Asp83 and Glu87 of procaspase-2 would be equivalent to Val69 and Asn73 in Apaf-1. Val69 is partially buried in Apaf-1 CARD and may not make interfacial contacts, but Asn73 is solvent accessible and mutation to alanine significantly decreased procaspase-9 CARD binding in our studies. These data suggest that procaspase-2 and Apaf-1 CARDs may use the same interface to interact. The only surface mutation reported for RAIDD that disrupts procaspase-2 binding is $\mathrm{G} 65 \mathrm{R}^{6}$; Gly65 is part of a tight loop that connects helices $\mathrm{H} 4$ and $\mathrm{H} 5$ in RAIDD CARD. While Gly65 may be part of the interaction interface it is also possible that the fold has been disrupted by this mutation. Many of the other mutations that have been reported to perturb the function of CARDs have been made to buried residues that are likely to disrupt the fold, and are therefore not informative. ${ }^{6}$ However, a point mutant in Nod1/CARD4, a recently identified Apaf- 1 homologue that contains an $\mathrm{N}$ terminal CARD and interacts with procaspase-9, is of interest. $^{24,25}$ Mutant Nod1 containing a V41Q substitution did not enhance caspase-9-induced apoptosis. ${ }^{25}$ Val41 in Nod1 is equivalent to Met26 in Apaf-1. While Met26 is buried in Apaf-1 CARD, and therefore a mutation here may disrupt the fold, it is also adjacent to Tyr24 and Asp27, residues identified in this study as mediating binding of procaspase-9 CARD. Combined with the conservation of the critical aspartic acid (equivalent to Asp27 in Apaf-1 CARD) these results suggest that Nod1 uses the same region to bind procaspase-9 CARD as Apaf-1 CARD.

Taken together these reports suggest that at least some CARDs may use the same interface as that identified in Apaf-1 CARD to bind other CARDs. Furthermore, several observations argue that some DEDs and CARDs use the same interaction interface. The DED of the adaptor FADD and the CARD of the adaptor molecule Apaf-1 are structurally very similar, and both interact with prodomains of caspases. The predicted interaction interface for the DED of FADD was based on a mutation ${ }^{17}$ that is analogous to Asp27, one of the most disruptive mutations in Apaf-1 CARD. In FADD DED the proposed interface included several exposed hydrophobic residues on helices $\mathrm{H} 2$ and H5. This extended area coincides with the interface defined by mutagenesis in this study, although in the case of Apaf1 CARD the residues are mostly charged.

Although it is likely that all CARDs, DDs and DEDs have similar folds and likely have similar interaction interfaces, they may not all interact in the same way. In the case of the interacting DDs from FADD and Fas it appears that the interaction is symmetric and involves residues on helices $\mathrm{H} 2$ and $\mathrm{H} 3$ from both molecules, ${ }^{18}$ while Apaf-1 CARD uses residues on helices $\mathrm{H} 2$ and $\mathrm{H} 3$ to interact with those on $\mathrm{H} 1$ and $\mathrm{H} 4$ in procaspase-9 CARD. Whether all DDs interact using similar residues and whether the asymmetry of the CARD interaction extends to other interacting pairs of domains remains to be determined. Elucidation of the three-dimensional structures of additional molecules, in 
association with site directed mutagenesis, will be crucial in delineating the interactions that generate selectivity.

\section{Materials and Methods}

\section{Preparation of Apaf-1 CARD NMR samples}

The CARD from human Apaf-1 (residues 1-97) was expressed in Escherichia coli BL21 (DE3) as a glutathione-S-transferase (GST) fusion protein using pGEX:6P-3 (Pharmacia). Protein was purified by affinity chromatography on glutathione Sepharose (Pharmacia) then cleaved with PreScission protease (Pharmacia) while bound to the resin. After cleavage five additional $\mathrm{N}$-terminal vector derived residues (GPLGS) remained. Soluble Apaf-1 CARD was further purified over a Superdex-75 column (Pharmacia). Purity was confirmed by SDSPAGE and electrospray mass spectrometry. Isotopically labelled proteins were isolated from cells grown on M9 medium containing ${ }^{15} \mathrm{NH}_{4} \mathrm{Cl}$ or ${ }^{15} \mathrm{NH}_{4} \mathrm{Cl}$ and $\left[\mathrm{U}-{ }^{13} \mathrm{C}\right]$-glucose. NMR samples contained $\sim 1.5 \mathrm{mM}$ protein in $20 \mathrm{mM}$ sodium phosphate $(\mathrm{pH} 6.7), 75 \mathrm{mM} \mathrm{NaCl}$, $2 \mathrm{mM}$ dithiothreitol (DTT) and $0.04 \%$ sodium azide in $\mathrm{H}_{2} \mathrm{O}:{ }^{2} \mathrm{H}_{2} \mathrm{O}(9: 1)$. Additional DTT was added to the NMR samples prior to acquisition of spectra to prevent dimer formation.

\section{NMR spectroscopy and spectral assignments}

NMR spectra were acquired at $30^{\circ} \mathrm{C}$ using Bruker DRX-600 and AMX500 spectrometers equipped with triple-resonance pulsed-field gradient probes. Sequential resonance assignments were made using a series of triple resonance spectra acquired on either uniformly ${ }^{15} \mathrm{~N}$ - or ${ }^{13} \mathrm{C},{ }^{15} \mathrm{~N}$-labelled protein. ${ }^{20}$ A $2 \mathrm{D}{ }^{1} \mathrm{H}^{-15} \mathrm{~N}-\mathrm{HSQC}, 3 \mathrm{D}{ }^{1} \mathrm{H}^{-15} \mathrm{~N}$ TOCSY-HSQC with a $30 \mathrm{~ms}$ isotropic mixing time, a $3 \mathrm{D}{ }^{1} \mathrm{H}-15 \mathrm{~N}$ NOESY with a 150 ms mixing period, HNHA, HNHB and heteronuclear $\left\{{ }^{1} \mathrm{H}\right\}-{ }^{15} \mathrm{~N}-\mathrm{NOE}$ experiments were acquired on ${ }^{15} \mathrm{~N}$-labelled Apaf-1 CARD. Constant time HNCA, HNCO, HNCACB and CBCA(CO)NH experiments ${ }^{20}$ and a 150 ms mixing time ${ }^{13} \mathrm{C}$-edited NOESY-HSQC were acquired on ${ }^{13} \mathrm{C},{ }^{15} \mathrm{~N}$-labelled Apaf-1. The ${ }^{13} \mathrm{C}$-edited NOESYHSQC was of sufficient quality to allow the direct assignment of side chain ${ }^{13} \mathrm{C}$ resonances. 2D NOESY spectra with 150 and 75 ms mixing times were recorded on unlabelled protein. Exchange properties of the $\mathrm{NH}$ protons were monitored by dissolving lyophilized ${ }^{15} \mathrm{~N}$-Apaf-1 in ${ }^{2} \mathrm{H}_{2} \mathrm{O}$ and acquiring a series of ${ }^{15} \mathrm{~N}-\mathrm{HSQC}$ spectra over a period of $20 \mathrm{~h}$. All $\mathrm{NHs}$ had exchanged after this period. Spectra were processed using XWIN-NMR (Bruker AG) and analyzed using XEASY. ${ }^{26}$

\section{Structure calculation}

Approximate inter-proton distances were derived from 2D-NOESY and $3 \mathrm{D}{ }^{13} \mathrm{C}$ - and ${ }^{15} \mathrm{~N}$-edited NOESY spectra. Bðackbone $\phi$ constraints were determined from ${ }^{3} \mathrm{~J}_{\mathrm{HNH} \alpha}$ coupling constants measured from a $\mathrm{HNHA}$ spectrum and side chain coupling constants, ${ }^{3} \mathrm{~J}_{\mathrm{N} \cdot \mathrm{H} \beta}$ determined from an HNHB spectrum. $\psi$ Angles were restricted according to the value of the chemical shift of their $\mathrm{C} \alpha$ resonance. ${ }^{27}$ Residues not restricted according to their ${ }^{3} \mathrm{~J}_{\mathrm{HNH} \alpha}$ coupling constant were restricted in their $\phi$ angle according to Luginbühl et al., ${ }^{27}$ or to negative $\phi$ angles where the condition for a positive $\phi$ angle was not met. ${ }^{28}$ Hydrogen bond constraints were applied where there existed a slowly exchanging $\mathrm{NH}$ proton, a $\phi$ angle restricted to the helical region and either a $d_{\alpha \beta}(i, i+3)$ or a $d_{\alpha N}(i, i+4)$ NOE present. ${ }^{29}$ Structure calculations were performed using DYANA ${ }^{30}$ and a similar protocol to that described previously. ${ }^{29}$ Once the final set of restraints had been obtained a new family of structures was generated using X-PLOR $(3.851)^{31}$ and refined using dynamic simulated annealing. The 50 lowest penalty function structures were selected from a calculation of 250 and minimized in a box of water. ${ }^{32}$ Structural analysis was performed on the 20 structures with the lowest stereochemical energies and PROCHECK_NMR ${ }^{33}$ was used for assessment of their stereochemical quality. Structural figures were created using MOLMOL. ${ }^{34}$ Coordinates have been submitted to the Protein Data Bank (accession no. 1CWW).

\section{Mutagenesis of Apaf-1 CARD and expression of procaspase-9 CARD}

Human caspase-9 CARD (residues 1-99) was expressed and purified as described for Apaf-1 CARD. A PCR based strategy was used to generate Apaf-1 CARD cDNA mutants. ${ }^{35}$ Double mutants were generated by performing a second round of mutagenesis using template containing one mutation. All constructs were verified by DNA sequencing. Each mutant was expressed as described for the wildtype protein except the GST fusion protein was not cleaved. Instead, all mutant GST fused proteins were immobilized on glutathione Sepharose then the resin was washed extensively and the amount of bound fusion protein estimated by SDS-PAGE. For binding experiments equivalent amounts of bound fusion protein (wt GST/ Apaf-1 CARD and GST/Apaf-1 CARD mutants) were aliquoted and a standard amount of cleaved soluble caspase-9 CARD was added. Volumes were made up to $25 \mu$ l with $1 \times$ PBS $(11.8 \mathrm{mM}$ phosphate buffer $\mathrm{pH}$ 7.3, $2.7 \mathrm{mM} \mathrm{KCl}, 140 \mathrm{mM} \mathrm{NaCl}, 1 \mathrm{mM}$ DTT) and mixed at room temperature for $30 \mathrm{~min}$. The resin was pelleted, supernatant removed, washed twice with $200 \mu \mathrm{l}$ of $1 \times$ PBS containing $0.02 \%$ Tween-20 and SDS-PAGE loading buffer was added before samples were analyzed by SDS-PAGE.

\section{Acknowledgements}

We thank David Huang for providing Apaf-1 and caspase-9 cDNAs; John Silke and Ray Norton for helpful discussions; Stan Moore for critically reading the manuscript. This work was supported in part by a grant from the Australian Government (NH\&MRC RegKey 9730002) to DL Vaux, the $\mathrm{CRC}$ for Cellular Growth Factors and a grant to CL Day from the Marsden Fund (New Zealand).

\section{References}

1. Vaux DL and Korsmeyer SJ (1999) Cell death in development. Cell 96: 245-254

2. Nicholson DW and Thornberry NA (1997) Caspases: killer proteases. Trends Biochem. Sci. 22: 299-306

3. Kumar S and Colussi PA (1999) Prodomains-adaptors-oligomerisation: the pursuit of caspase activation in apoptosis. Trends Biochem. Sci. 24: 1-4

4. Hofmann K, Bucher P and Tschopp J (1997) The CARD domain: a new apoptotic signalling motif. Trends Biochem. Sci. 22: 155-156

5. Boldin MP, Goncharov TM, Goltsev YV and Wallach D (1996) Involvement of $\mathrm{MACH}$, a novel MORT1/FADD-interacting protease, in Fas/APO-1- and TNF receptor-induced cell death. Cell 85: 803-815

6. Li P, Nijhawan D, Budihardjo I, Srinivasula SM, Ahmad M, Alnemri ES and Wang $X$ (1997) Cytochrome $c$ and dATP-dependent formation of Apaf-1/caspase-9 complex initiates an apoptotic protease cascade. Cell 91: 479-489

7. Duan H and Dixit VM (1997) RAIDD is a new 'death' adaptor molecule. Nature (London) 385: 86-89

8. Cecconi F, Alvarez-Bolado G, Meyer BI, Roth, KA and Gruss P (1998) Apaf1 (CED-4 homolog) regulates programmed cell death in mammalian development. Cell 94: 727-737 
9. Hakem R, Hakem A, Duncan GS, Henderson JT, Woo M, Soengas MS, Elia A, de la Pompa JL, Kagi D, Khoo W, Potter J, Yoshida R, Kaufman SA, Lowe SW, Penninger, JM and Mak TW (1998) Differential requirement for caspase 9 in apoptotic pathways in vivo. Cell 94: 339-352

10. Kuida K, Haydar TF, Kuan CY, Gu, Y, Taya C, Karasuyama H, Su MSS, Rakic P and Flavell RA (1998) Reduced apoptosis and cytochrome c-mediated caspase activation in mice lacking caspase 9. Cell 94: 325-337

11. Yoshida H, Kong YY, Yoshida R, Elia, AJ, Hakem A, Hakem R, Penninger JM and Mak TW (1998) Apaf1 is required for mitochondrial pathways of apoptosis and brain development. Cell 94: 739-750

12. Srinivasula SM, Ahmad M, Fernandes-Alnemri T and Alnemri ES (1998) Autoactivation of procaspase-9 by Apaf-1-mediated oligomerization. Mol. Cell 1: 949-957

13. Zou H, HenzelWJ, Liu XS, Lutschg A and Wang X (1997) Apaf-1, a human protein homologous to $C$. elegans CED-4, participates in cytochrome c-dependent activation of caspase-3. Cell 90: 405-413

14. Huang B, Eberstadt M, Olejniczak ET, Meadows RP and Fesik SW (1996) NMR structure and mutagenesis of the Fas (APO-1/CD95) death domain. Nature (London) 384: 638-641

15. Leipinsh E, llag LI, Otting $G$ and Ibanez CF (1997) NMR structure of the death domain of the p75 neurotrophin receptor. EMBO J. 16: 4999-5005

16. Chou JJ, Matsuo H, Duan H and Wagner G (1998) Solution structure of the RAIDD CARD and model for CARD/CARD interaction in caspase-2 and caspase-9 recruitment. Cell 94: 171-180

17. Eberstadt M, Huang B, Chen Z, Meadows RP, Ng SC, Zeng L, Lenardo MJ and Fesik SW (1998) NMR structure and mutagenesis of the FADD (Mort1) deatheffector domain. Nature (London) 392: 941-945

18. Jeong EJ, Bang S, Lee TH, Park YI, Sim WS and Kim KS (1999) The solution structure of FADD death domain. J. Biol. Chem. 274: 16337-16342

19. Qin H, Srinivasula SM, Wu G, Fernandes-Alnemri T, Alnemri ES and Shi Y (1999) Structural basis of procaspase-9 recruitment by the apoptotic proteaseactivating factor 1. Nature (London) 399: $549-557$

20. Bax A and Grzesiek S (1993) Methodological advances in protein NMR. Acc. Chem. Res. 26: 131-138

21. Lu G (1996) A WWW service system for automatic comparison of protein structures. Protein Data Bank Quarterly Newsletter 78: 10-11

22. Bass SH, Mulkerrin MG and Wells JA (1991) A systematic mutational analysis of hormone-binding determinants in the human growth hormone receptor. Proc. Natl. Acad. Sci. USA 88: 4498-4502
23. Hu Y, Ding L, Spencer DM and Núñez G (1998) WD-40 repeat region regulates Apaf-1 self-association and procaspase-9 activation. J. Biol. Chem. 273: 33489-33494

24. Bertin J, Nir WJ, Fischer CM, Tayber OV, Errada PR, Grant JR, Keilty JJ, Gosselin ML, Robison KE, Wong GHW, Glucksmann MA and DiStefano PS (1999) Human CARD4 protein is a novel CED-4/Apaf-1 cell death family member that activates NF-kappaB. J. Biol. Chem. 274: 12955-12958

25. Inohara N, Koseki T, Chen S, del Peso L, HuY, Yee C, Chen S, Carrio R, MerinoJ, Liu D, Ni J and Núñez G (1999) Nod1, an Apaf-1-like activator of caspase-9 and nuclear factor- $k$ B. J. Biol. Chem. 274: 14560-1456721.

26. Bartels C, Xia TH, Billeter M, Güntert $P$ and Wüthrich K (1995) The program XEASY for computer-supported NMR spectral-analysis of biological macromolecules. J. Biomol. NMR 6: 1-10

27. Luginbühl P, Szyperski T and Wüthrich K (1995) Statistical basis for the use of ${ }^{13} \mathrm{C}\{\mathrm{STXT}\} \alpha\{X T X T\}$ chemical shifts in protein structure determination. J. Magn. Reson. Ser. B 109: 229-233

28. Ludvigsen S and Poulsen FM (1992) Positive $\phi$-angles in proteins by nuclear magnetic resonance spectroscopy. J. Biomol. NMR 2: 227-233

29. Hinds MG, Maurer T, Zhang JG, Nicola NA and Norton RS (1998) Solution structure of leukemia inhibitory factor. J. Biol. Chem. 273: 13738-13745

30. Güntert P, Mumenthaler C and Wüthrich K (1997) Torsion angle dynamics for NMR structure calculation with the new program DYANA. J. Mol. Biol. 273: 283298

31. Brünger A (1992) X-PLOR. A System for X-ray crystallography and NMR. (Yale University, New Haven, $C T$ )

32. Linge JP and Nilges M (1999) Influence of non-bonded parameters on the quality of NMR structures: A new force field for NMR structure calculation. J. Biomol. NMR 13: $51-59$

33. Laskowski RA, Rullmann JAC, Macarthur MW, Kaptein R and Thornton JM (1996) AQUA and PROCHECK-NMR: programs for checking the quality of protein structures solved by NMR. J. Biomol. NMR 8: 477-486

34. Koradi R, Billeter M and Wüthrich K (1996) MOLMOL: a program for display and analysis of macromolecular structures. J. Mol. Graphics 14:51-55

35. Ho SN, Hunt HD, Horton RM, Pullen JK and Pease LR (1989) Site-directed mutagenesis by overlap extension using the polymerase chain reaction. Gene 77: $51-59$ 